Tohoku J. Exp. Med., 2012, 228, 9-16

\title{
Accurate Preoperative Staging of Gastric Cancer with Combined Endoscopic Ultrasonography and PET-CT
}

\author{
Baiwen Li, ${ }^{1 *}$ Ping Zheng, ${ }^{1 *}$ Qi Zhu' ${ }^{2}$ and Jun Lin $^{3}$ \\ ${ }^{1}$ Department of Gastroenterology, Shanghai First People's Hospital, School of Medicine, Shanghai Jiaotong \\ University, Shanghai, P.R. China \\ ${ }^{2}$ Department of Gastroenterology, Ruijin Hospital, School of Medicine, Shanghai Jiaotong University, Shanghai, \\ P.R. China \\ ${ }^{3}$ Department of Pathology, Shanghai First People's Hospital, School of Medicine, Shanghai Jiaotong University, \\ Shanghai, P.R. China
}

Accurate staging of gastric cancer is helpful to determine the most appropriate therapy, but no staging modality has been accepted as the standard. Objective is to evaluate the usefulness of endoscopic ultrasonograph (EUS) combined with position emission tomography and computed tomograph (PET-CT) in gastric cancer staging. A total of 124 patients confirmed with gastric cancer were subjected to staging with EUS and PET-CT scanning. The detection rate of primary tumor was $99.2 \%$ by combination use of two modalities and $97.6 \%$ by EUS alone $(p=0.6219)$, but the detection rate was $90.3 \%(112$ of 124$)$ by PET-CT alone (compared with the combination, $p=0.0027$; compared with EUS alone, $p=0.0299$ ). The locoregional lymph node invasion was identified in $84 / 124(67.7 \%)$ by combined PET-CT and EUS, which was obviously higher than that with EUS $(52.4 \%)$ or PET-CT $(43.5 \%)$ alone $(p=0.0194$ and $p=0.0002$, respectively). There was no statistical difference in identification of celiac axis lymph node metastasis among three methods, but the combined examination or PET-CT alone was more effective than EUS alone in the detection of distant metastases (all $p<0.01$ ). Furthermore, the combined EUS and PET-CT was more optimal than using EUS or PET-CT alone in the accurate $\mathrm{T}$ and $\mathrm{N}$ staging and the effect on change of treatment. The present study indicates that the combination of EUS and PET-CT is an ideal modality in the preoperative staging of gastric cancer and it provides beneficial guidance for the treatment of gastric cancer.

Keywords: computed tomography; endoscopic ultrasound; gastric cancer; positron emission tomography; preoperative staging

Tohoku J. Exp. Med., 2012 Sep, 228 (1), 9-16. 일 2012 Tohoku University Medical Press

Gastric cancer is the fourth most common cancer and the second leading cause of cancer mortality (Parkin et al. 2002; Kamangar et al. 2006). Only complete resection of all gross disease with negative microscopic margins (R0 resection) provides a long-term survival benefit, and even then the overall 5-year relative survival rate is approximately 20\% (Martin et al. 2002; Kamangar et al. 2006). The effectiveness of surgery as the sole treatment for advanced locoregional disease has been challenged, and considerable effort is currently devoted to evaluating the benefit of adjuvant and neo-adjuvant therapies for improving patient survival (O'Reilly and Forastiere 1995). Until recently, precise preoperative staging in gastric cancer was not essential because knowledge of the cancer stage did not alter treatment plans. However, with recent therapeutic advances, the accurate preoperative assessment of gastric cancer staging is increasingly important for the appropriate selection of patients for specific treatment regimens. For example, in patients with early gastric cancer, especially in cases confined to mucosa, endoscopic resection is performed to avoid unnecessary surgical procedures (Gotoda 2006).

However, despite the importance of pre-treatment staging, no single or combination of tests has been accepted as standard for the staging of gastric cancer. Current practice varies and may include the use of computed tomography (CT), positron emission tomography (PET), and endoscopic ultrasound (EUS) either alone or in various combinations in the preoperative staging process. EUS can provide tumor $(\mathrm{T})$ staging, identify local lymph node $(\mathrm{N})$ staging, and provide a diagnosis of malignant lymph node invasion when used in conjunction with fine needle aspira-

Received May 31, 2012; accepted July 21, 2012. Published online August 4, 2012; doi: 10.1620/tjem.228.9.

*The authors Baiwen Li and Ping Zheng contributed equally to this work.

Correspondence: Baiwen Li, Department of Gastroenterology, Shanghai First People's Hospital, School of Medicine, Shanghai Jiaotong

University, Shanghai 200080, P.R. China.

e-mail: muzibowen@126.com 
tion (FNA). However, EUS cannot be used for the detection of metastatic disease (Meltzer et al. 2000)

CT scans have traditionally been used for metastatic staging, while PET scanning is gaining in popularity for gastric cancer staging. The combined use of PET and CT scanning is expected to offer a single test to identify possible metastases and determine if they are metabolically active. This technique has shown significant promise in esophageal cancer (Walker et al. 2011). However, to date, PET-CT has not been evaluated in gastric cancer staging, particularly in conjunction with EUS.

The present study was conducted to evaluate the significance of EUS combined with PET-CT in the accurate staging of gastric cancer prior to treatment. Meanwhile, the study also investigates the impact that the outcome of EUS and PET-CT scanning has on the treatments offered to patients with gastric cancer. The results indicate that the combination of EUS and PET-CT is an ideal modality in the staging of gastric cancer and provides beneficial guidance for the treatment of gastric cancer.

\section{Materials and Methods}

\section{Study design}

Overall 124 patients of consecutive biopsy-proven gastric cancer at the First People's Hospital, Shanghai JiaoTong University between March 2007 and September 2011 who underwent surgical resection or endoscopic resection were included in this study. The study has been approved by the Ethics Committee of the First People's Hospital in accordance with the Helsinki Declaration, and written informed consents were obtained from all patients.

Cancer staging was performed based on the combination use of EUS and PET-CT scanning. The results were interpreted by two gastroenterologists who performed the EUS and two nuclear medicine physicians, independently, in a non-blinded fashion. Subsequent patient management was recorded (primary surgical resection, surgical resection after neo-adjuvant therapy with chemoradiation, surgical therapy followed by adjuvant chemoradiation, chemoradiation alone, or strictly palliative therapy).

\section{EUS}

After diagnosis was confirmed, EUS was performed using a radial scanning ultrasonic endoscope (GF-UE260-AL5/GF-UC240PAL5; Olympus, Tokyo, Japan). Two experienced endoscopists carried out all procedures following intravenous administration of scopolamine butylbromide $(20 \mathrm{mg})$. After evaluation of the primary lesion, water was infused into stomach (approximately 200-500 mL) to examine the five-layered structure of gastric wall. The $\mathrm{T}$ staging was then recorded: T1, invasion into the mucosa or submucosa; T2, invasion into muscularis propria; T3, invasion into adventitia; and T4, invasion into adjacent structures, as well as the presence or absence of regional lymph node metastases (N0 or N1) and celiac axis nodes (CAX). Lymph node EUS features were used to determine the likelihood of malignancy, with a larger size $(>10 \mathrm{~mm})$ considered to increase the chance of malignancy but not considered an absolute prerequisite for malignancy. FNA was performed at the time of EUS if this was technically feasible, if the needle would not traverse the primary tumor, or if the results would alter subsequent management.

\section{PET-CT}

Patients fasted for at least 4 hours prior to the intravenous administration of approximately $10 \mathrm{mCi}$ of 2-deoxy-2-[18F]fluoro-Dglucose (FDG; adjusted for weight). After resting for approximately 45-60 minutes, patients were asked to void before entering the PET-CT scanner (GE Discovery LS PET-CT scanner) with arms down at their side for a dose, non-contrast CT scan $(120 \mathrm{~mA}, 140 \mathrm{kV}$, $5 \mathrm{~mm}$ slices) which was used for attenuation correction and signal localization. A FDG-PET scan procedure was then performed from the skull base to the thighs for 5 min per bed position. Pictures were viewed in the transaxial, coronal, and sagittal planes, with images coregistered with the CT scan images when needed.

\section{Determining change in treatment}

All patients were initially regarded candidates for surgery, and the decisive management was altered as a result of a staging test. The presence of distant metastases or invasion into local structures which precluded curative surgical resection was also considered as a change in treatment. Patients who ultimately did not undergo surgery for reasons not connected to the preoperative imaging were excluded from the analysis.

\section{Determining tumor and nodal staging accuracy}

Pathology staging data were obtained for all patients $(n=54)$ who underwent primary surgical resection without prior chemoradiation that could affect the stage of the cancer. The staging of the tumor and nodes in these patients represented the standard against which to compare staging with EUS and PET-CT.

\section{Statistical analysis}

Classification of the locoregional adenopathy and celiac axis nodes into normal and abnormal categories according to EUS and PET-CT were compared. McNemar's test was used to assess the difference in the percentage success in the modalities.

\section{Results}

\section{Clinical characteristics}

Overall 124 patients (92 men; 32 women) presenting with biopsy-proven adenocarcinoma of the stomach between March 2007 to September 2011 were included in the study. The distribution of gross and clinicopathological features is listed in Table 1.

\section{Identification of primary tumor}

Among 124 patients, primary tumors were identified in 121 patients (97.6\%) by EUS and in 123 patients $(99.2 \%)$ by combined EUS and PET-CT, whereas primary tumors were confirmed in 112 patients $(90.3 \%)$ by PET-CT alone. The exceptional patient by combined examination was due to one point cancer, in which cancer cells were detected with microscopic examination of endoscopic biopsy specimens but they were not detected with a postoperative histopathologic examination. The difference were statistically significant when PET-CT compared with combined use of two modalities or EUS alone (PET-CT versus EUS, $p=$ 0.0299; PET-CT versus EUS plus PET-CT, $p=0.0027$ ). Although there was difference between EUS and combined two modalities, no statistical significance was observed ( $p$ 
Table 1. Gross and clinicopathological features.

\begin{tabular}{lc}
\hline \multicolumn{1}{c}{ Characteristic } & No. patients $(\%)$ \\
\hline Gender & \\
Male & $92(74.2)$ \\
Female & $32(25.8)$ \\
Location & \\
Antrum & $49(39.5)$ \\
Body & $38(30.6)$ \\
Angle & $24(19.4)$ \\
Fundus & $13(10.5)$ \\
Histology & \\
Well-differentiated tubular adenocarcinoma & $12(9.7)$ \\
Moderately-differentiated tubular adenocarcinoma & $55(44.4)$ \\
Poorly-differentiated adenocarcinoma & $38(30.6)$ \\
Signet-ring cell carcinoma & $15(12.1)$ \\
Mucinous adenocarcinoma & $4(3.2)$ \\
Size & \\
$\leq 2$ cm & $31(25)$ \\
$2 \sim 4$ cm & $59(47.6)$ \\
$\geq 4$ cm & $34(27.4)$ \\
Stage & $21(15.9)$ \\
Early gastric cancer & $103(84.1)$ \\
Advanced gastric cancer &
\end{tabular}

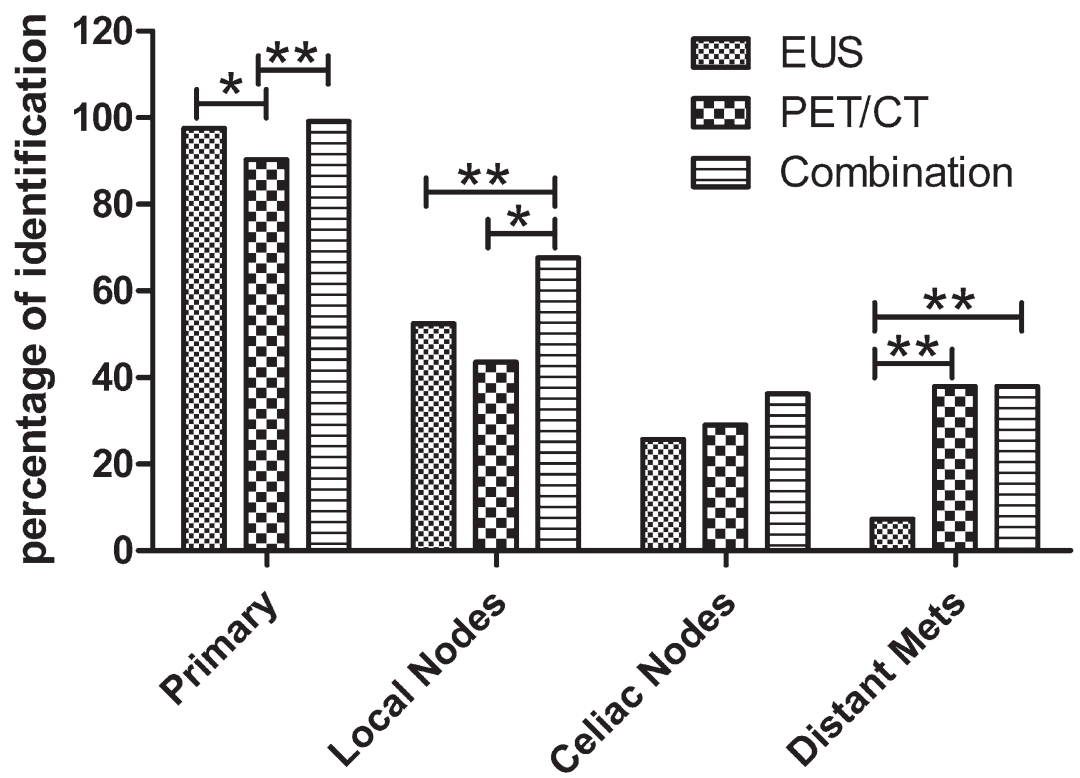

Fig. 1. The detection ability of three methods in TNM staging of gastric cancer.

Bar graph displaying relative ability of EUS, PET-CT and combination using two modalities to identify the primary gastric tumor, locoregional lymphadenopathy, celiac nodes and distant metastases expressed in the percentage of patients identified. The combination modality is obviously superior to EUS or PET-CT alone whether T, N or M staging. $* p<0.05 ; * p<0.01$.

$=0.6219)$ (Fig. 1). All twelve gastric carcinomas that were not detected by PET-CT were staged in T1 (Fig. 3). Overall, $19 \mathrm{~T} 1(15.7 \%), 23 \mathrm{~T} 2(19.0 \%), 68 \mathrm{~T} 3(56.2 \%)$, and 9 T4 (7.4\%) were staged by EUS, but 2 patients (1.7\%) could not be determined by EUS (Tx).

\section{Locoregional lymph node staging}

Sixty-five (52.4\%) patients were identified by EUS, 


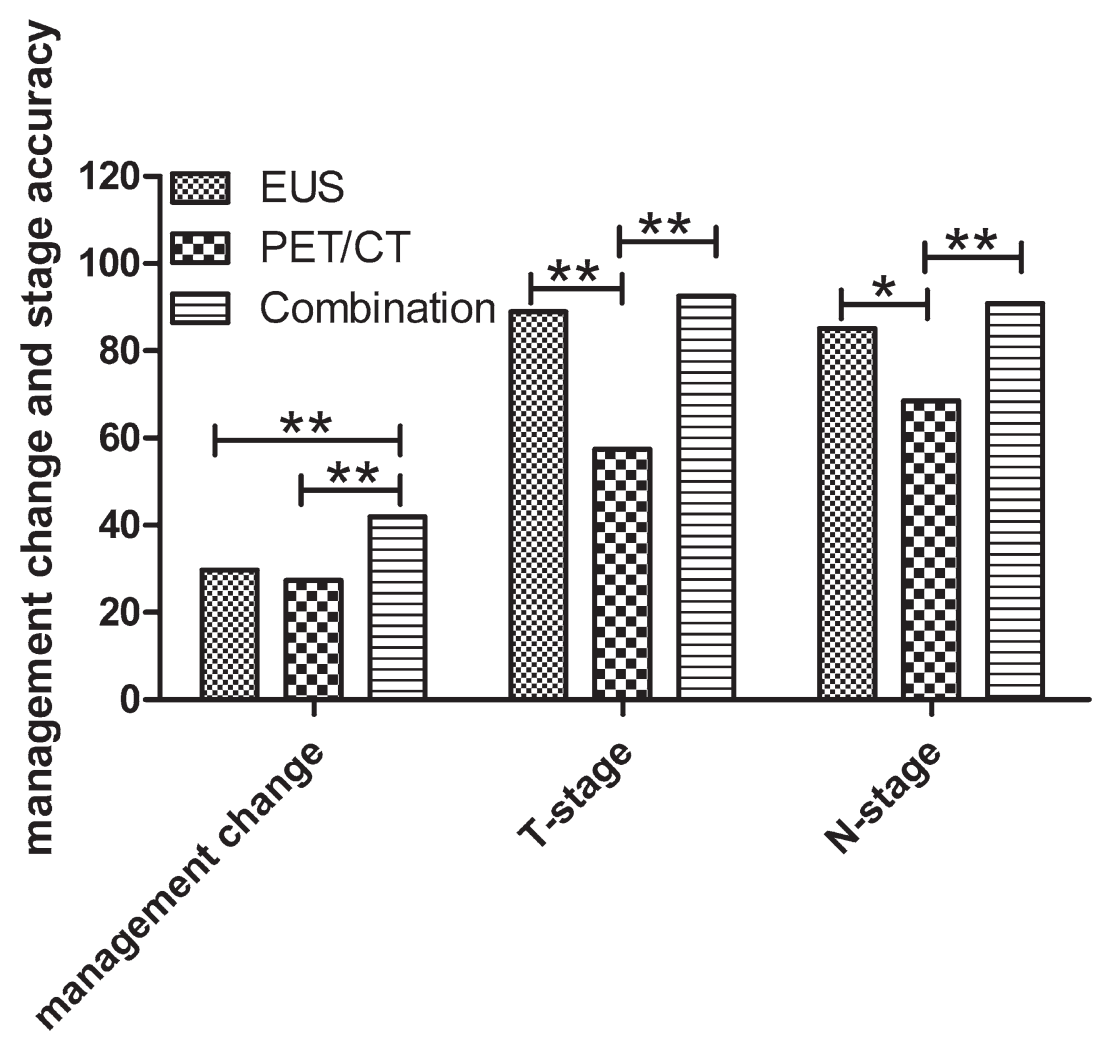

Fig. 2. The effect on treatment change and the accuracy rate of $\mathrm{T}$ or $\mathrm{N}$ staging identified by three methods.

The combination modality is obviously superior to EUS or PET-CT alone in changing the treatment of gastric cancer. The PET-CT is inferior to combination modality or EUS alone in identification of accuracy rate of T or N staging. ${ }^{*} p<$ $0.05 ; * p<0.01$.

while 54 (43.5\%) were confirmed by PET-CT with regional lymph node invasion. The detected rate of locoregional node invasion was obviously increased when combined use of two modalities, 84 of 124 patients $(67.7 \%)$ were identified (EUS versus EUS plus PET-CT, $p=0.0194$; PET-CT versus EUS plus PET-CT, $p=0.0002$ ) (Fig. 1). Four patients with locoregional node invasion were identified by PET-CT but not by EUS, while 26 patients by EUS protocol but not by PET-CT (Fig. 4). EUS-FNA (EUS in conjunction with fine needle aspiration biopsy) was also performed in 37 patients with locoregional lymph node invasion.

\section{Identification of celiac axis nodes}

The celiac axis node is the position for metastasis of gastric cancer cells easily and identified conveniently by EUS, and so we detected this location as a study target. A total of $32(25.8 \%), 36(29.0 \%)$ and $45(36.3 \%)$ patients with celiac axis lymph invasion were identified by EUS, PET-CT and combination of above two modalities, respectively. There was no statistically significant difference among three modalities $(p>0.05)$ (Fig. 1). EUS and PET-CT agreed in 28 patients on the presence of CAX nodes and in 55 patients on the absence of CAX nodes. In one patient PET-CT identified a celiac node, but the EUS excluded. EUS did not successfully assess celiac axis nodes in 19 patients because endoscope could not traverse the primary tumor, but celiac axis nodes were identified by PET-CT in three of these patients (Fig. 5).

\section{Identification of distant metastases}

Forty-seven of $124(37.9 \%)$ patients were confirmed with metastases by PET-CT (liver, $n=18$; left supraclavicular lymph nodes, $n=12$; peritoneal cavity, $n=9$; pelvic cavity, $n=5$; lung, $n=3$ ). The results of combined use of two modalities were similar to those of PET-CT alone in identification of distant metastases. But EUS identified only 9 of $124(7.3 \%)$ T4 patients with tumor invasion into the liver (Fig. 1). Figure 6 shows an example PET-CT scanning of a patient with metastatic disease to the liver.

\section{Change in management}

Detection of metastatic disease through PET-CT led to prescription for chemoradiation therapy or palliative care in 34 of 124 instances $(27.4 \%)$. EUS changed patients care to neo-adjuvant therapy prior to surgical resection in 37 of 124 cases $(29.8 \%)$. The population of cases changed in management by combination of two modalities was significantly increased, 52 of $124(41.9 \%)$ cases benefit from the change ( $p<0.01$, compared with EUS or PET-CT) (Fig. 2).

\section{Accuracy of tumor and node staging}

Surgical pathology staged 19 T1 (35.2\%), 21 T2 

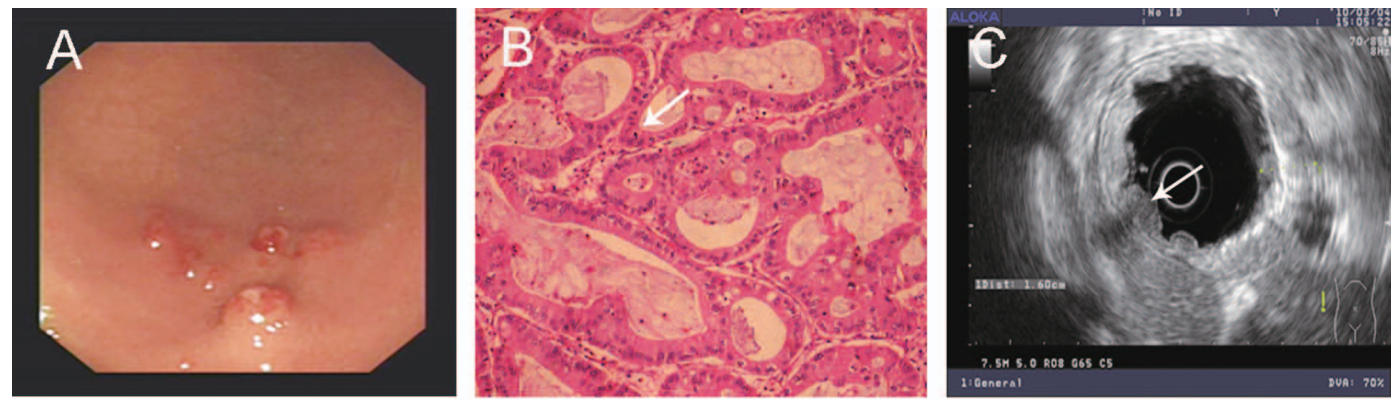

Fig. 3. An example for T staging.

A 52-year-old male patient, was identified early gastric cancer by EUS, but was not detected by PET-CT imaging. A, endoscopy found protrusion lesion in the greater curvature of body of stomach; B, histopathology proved well differentiation adenocarcinoma. HE staining $(\times 100)$ indicate the cancer cells shaped in cube or column, and arranged into gland tubular structure with nearly consistent size (white arrow); C, EUS showed lesion was confined mucous layer (white arrow).
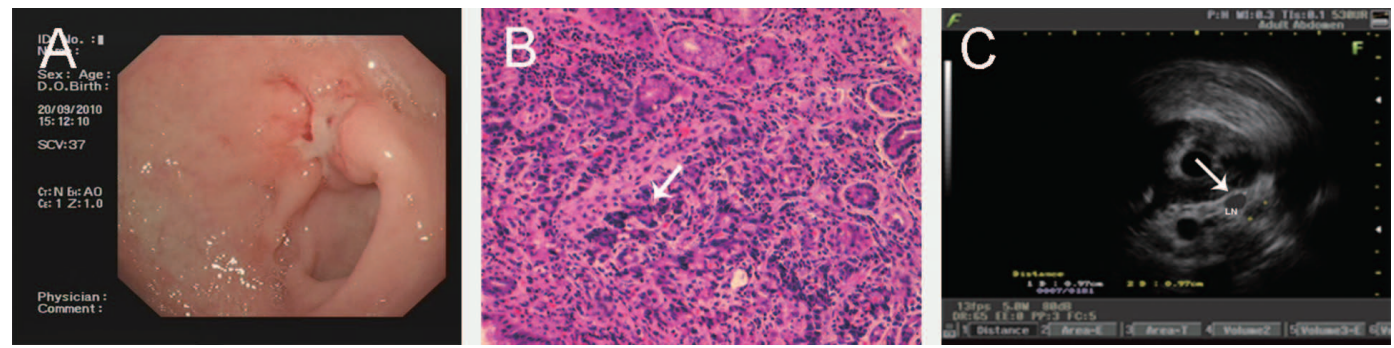

Fig. 4. An example for $\mathrm{N}$ staging.

A 65-year-old female patient, was identified N1 stage gastric cancer by EUS, but was not detected by PET-CT imaging. A, endoscopy found an irregular ulcer in anterior wall of antrum; B, histopathology proved poor differentiation adenocarcinoma. HE staining $(\times 100)$ indicate cancer cells shaped in short-column or unshapement, and with small-nest or cord-like arrangement, almost without gland tubular structure (white arrow); C, EUS showed the layers of gastric wall disappeared and an abnormal regional node was detected (white arrow).
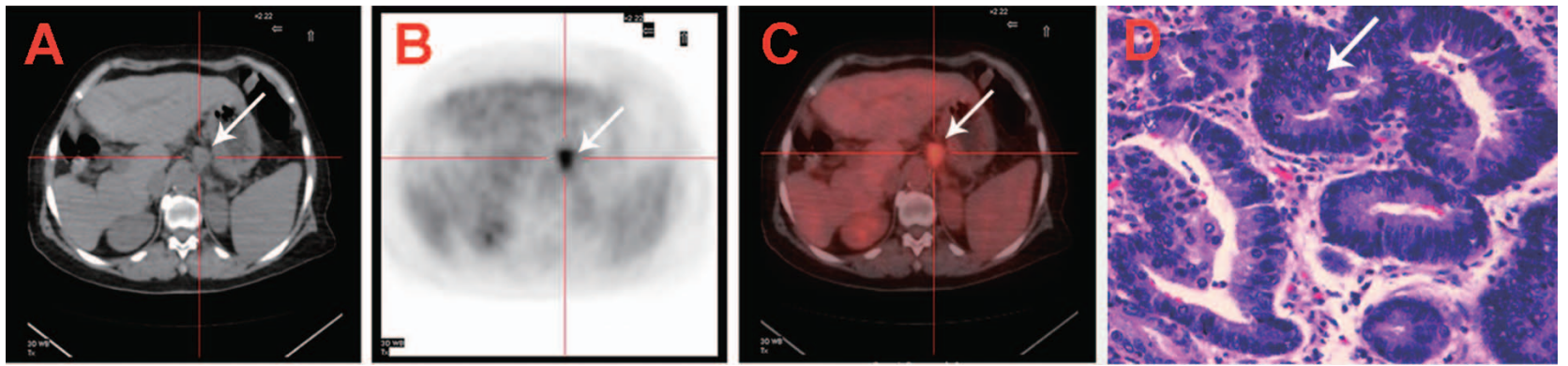

Fig. 5. Detection of celiac axis lymph node metastasis

PET-CT image of a patient with celiac lymphadenopathy that was detected solely by PET-CT (white arrows) as EUS was unable to traverse the primary tumor. A, CT transaxials; B, PET transaxials; C, fused transaxials; and D, HE staining $(\times 200)$ indicated the morphology and size of glands were very inconsistent, epithelial cells were arranged in disorder and multilayer, nuclei was increased obviously, abnormity and accompanied by multiple karyokinesis (white arrow).

(38.9\%), and $14 \mathrm{~T} 3(25.9 \%)$ tumors while EUS staged 18 $\mathrm{T} 1$ (33.3\%), $19 \mathrm{~T} 2(35.2 \%)$ and $11 \mathrm{~T} 3(20.1 \%)$ tumors among the 54 patients who underwent surgery without antecedent chemotherapy. The accuracy rate of EUS in T staging was $88.9 \%(48 / 54)$ in this subset, and the PET-CT alone as well as the combination was $57.4 \%(31 / 54)$ and $92.6 \%$ (50/54), respectively. There were statistical differences when PET-CT compared with combined use of two modali- ties or EUS alone (all $p<0.01$ ). Among 54 patients who underwent primary resection, 22 patients were confirmed with regional lymph node metastasis and 32 patients without. The agreed rate of nodal staging by EUS, PET-CT, and the combined two modalities with the surgical pathology data was $87 \%$ (47/54), 68.5\% (37/54), and 90.8\% (49/54), respectively. The differences between PET-CT and the combination tests or EUS alone in $\mathrm{N}$ staging accuracy were 

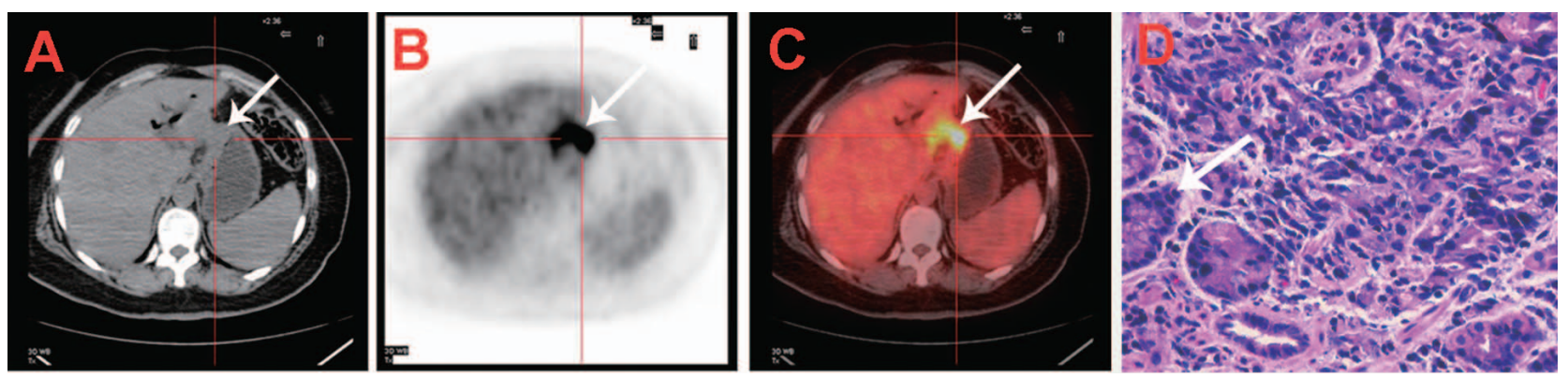

Fig. 6. Detection of distant metastases

Advanced gastric cancer in a 62-year-old man. PET-CT image of a patient with metastatic disease to the liver (white arrows). A, CT transaxials; B, PET transaxials; C, fused transaxials; and D, HE staining $(\times 200)$ indicated the epithelial cells were arranged in disorder and multilayer, the nuclei was increased obviously and accompanied by abnormity (white arrow).

obviously significant $(p=0.0035$ and $p=0.0076$, respectively). However, there was no statistically significant difference between EUS and the combined use of two modalities in the accuracy of $\mathrm{T}$ staging and $\mathrm{N}$ staging (Fig. 2).

\section{Discussion}

Gastric cancer is one of the most common malignant tumors of the alimentary tract. According to the reports, the incidence rate of gastric cancer in China ranks the highest among all malignant tumors. Every year, about 300,000 people are diagnosed with gastric cancer in China, accounting for approximately $33 \%$ of total confirmed patients in the world. About 260,000 people die of gastric cancer annually, accounting for $23 \%$ of deaths due to malignant cancer, and the number is still rising (Terry et al. 2002). To date, the treatment for intermediate and advanced gastric cancer is still a comprehensive surgery-centered strategy. Therefore, improved early diagnosis, accurately clinical staging, and optimal surgical procedures are essential to improve prognosis.

Use of EUS can clearly reveal the relationship between the structure of the alimentary tract wall and its adjacent organs. Since its development in the 1980s, EUS has been applied in the accurate assessment of the depth of tumor invasion as well as in the exposure of the regional lymph nodes and adjacent organs. This contributes to disease estimation, selection of surgical procedure and prognosis assessment (Willis et al. 2000; Kwee and Kwee 2007). With constant improvements in EUS, its superiority in T and $\mathrm{N}$ staging of gastric cancer has been demonstrated (Greenberg et al. 1994; Hizawa et al. 2002; Akashi et al. 2006). However, due to the limitation of the probe frequency of endoluminal sonography and the penetration depth of the ultrasonic beam, its inferiority in $\mathrm{M}$ staging of gastric cancer (i.e., its incapacity to accurately predict the distant metastasis of gastric cancer) is also shown (Ziegler et al. 1993; Bhandari et al. 2004; Chen et al. 2007).

With the development of medical imaging technology, multi-level spiral CT and MRI have been employed in preoperative staging and postoperative follow-up, although with low accuracy rate. The reported accuracy rate is approximately 51\%-59\% (Dux et al. 1999; Sohn et al. 2000), and the staging of early gastric cancer is far from satisfactory. Newly developed PET-CT technology using computer software can fuse the PET metabolic-change image with the 3D image of the corresponding anatomical location in the CT image, thus achieving both qualitative and positioning diagnosis in one examination (Townsend and Beyer 2002). Therefore, the diagnostic accuracy of cancer metastasis of the lymph nodes and organs distant from tumor is greatly improved. This study aimed to investigate the clinical value of T, N, M staging of gastric cancer with the combined use of EUS and PET-CT, and then study the influence of the results obtained by combined the two methods in the comprehensive treatment of gastric cancer.

In this study, a total of 124 patients were subjected to qualitative diagnosis of primary gastric cancer as well as $\mathrm{T}$ staging using a combination of EUS and PET-CT scanning. Compared with pathological histology, the overall accuracy rate of $\mathrm{T}$ staging was $92.6 \%(50 / 54)$, which was higher than by PET-CT or EUS alone (all $p<0.01$ ), and it is consistent with an earlier report by Tsendsuren et al. (63\%-88\%) (Chen et al. 2002; Tsendsuren et al. 2006; Ganpathi et al. 2006). Among 124 patients with primary gastric cancer, 115 patients were confirmed using PET-CT with a detection rate of $92.7 \%$, compared to $97.6 \%$ with EUS and $99.2 \%$ with the combination use of two modalities. The difference was of statistical significance between PET-CT and EUS or the combination tests ( $p<0.05$ and $p<0.01$, respectively). On the other hand, the twelve patients of missed diagnosis using PET-CT were all T1 cases, indicating that EUS is significantly superior to PET-CT in the diagnosis of early gastric cancer.

Normal lymph nodes are difficult to distinguish from regional fat and fibrous tissues due to their similar echo pattern in the EUS image. The identification of inflammatory swelling in the lymph nodes and metastatic lymph nodes is crucial to the EUS diagnosis of locoregional lymphadenopathy involvement in gastric cancer. The acknowledged standards for the diagnosis of metastatic lymph nodes are as 
follows (Catalano et al. 1994): (1) diameter $\geq 10 \mathrm{~mm}$; (2) round; (3) clear boundary; (4) hypo-echoic feature inside. According to the literature, the accuracy rate of EUS in $\mathrm{N}$ staging of gastric cancer fluctuates between $47 \%$ and $90 \%$ (Willis et al. 2000; Polkowski et al. 2004). Our research showed that the detection rate of locoregional lymph invasion by EUS and PET-CT was 52.4\% and 43.5\%, respectively. But it was increased to $67.7 \%$ when combined two modalities. A total of 54 cases was compared with the pathological results, the accuracy rate of combination of two modalities in $\mathrm{N}$ staging was $90.8 \%$, which was higher than that of EUS $(85.2 \%)$ or PET-CT $(68.5 \%)$. This result suggests that in the detection of regional metastatic lymph nodes, EUS is also superior to PET-CT, which might be due to the improvement of the devices used in EUS and the wide application of EUS-FNA. While the accuracy and specificity of the staging of metastatic lymph nodes can be further improved when combining EUS and PET-CT.

It should be acknowledged that due to the limitation of both the penetration depth of the ultrasonic beam (frequency 7.5 $\mathrm{MHz}$; maximum penetration depth $5-7 \mathrm{~cm}$ ) and the anatomical structure of stomach, EUS was inferior to PET-CT and combined examination in the diagnosis of distant metastasis of gastric cancer. Among 124 patients, only nine $(7.3 \%)$ were diagnosed with distant metastasis using EUS. In some patients, the diagnosis is actually impossible due to the small gastric cavity. By contrast, PET-CT combines the advantages of both anatomical positioning and energy-image-based qualitative determination, without the restraint of the conditions mentioned above. In this study, 47 patients $(37.9 \%)$ with metastasis were detected with PET-CT or with the combined examination, which is obviously higher than that of EUS alone.

In conclusion, PET-CT combines PET's sensitivity to $\mathrm{M}$ staging and CT's superiority to $\mathrm{N}$ staging, and the fused images generated can reveal not only anatomical changes, such as size, shape and density, but also metabolic dysfunction. Therefore, the detection rate of regional metastatic lymph nodes and the positioning and qualitative determination of metastasis can clearly be improved. However, PET-CT still confuses $\mathrm{T}$ staging and underestimates $\mathrm{N}$ staging. By contrast, EUS shows superiority in $\mathrm{T}$ staging in particular as well as $\mathrm{N}$ staging. With the application of EUS-FNA, EUS has a higher accuracy rate in $\mathrm{N}$ staging. However, the objective of our research was not to compare the respective sensitivity and accuracy of EUS and PET-CT in the preoperative staging of gastric cancer, but to integrate the respective advantages of EUS and PET-CT in staging of gastric cancer, in the hope that $\mathrm{T}, \mathrm{N}$ and $\mathrm{M}$ staging could achieve higher accuracy. Our results signified, whether in tumor detection, lymph node identification, presence of metastatic disease, or in effect on patient management, the combination of EUS and PET-CT scanning was all superior to respective procedure alone. In this study, 21 patients declined surgery upon the confirmation of distant metastasis, while another 41 received preoperative adjuvant che- motherapy with combining examination. Thus it provides beneficial guidance for the treatment of gastric cancer.

To the best of our knowledge, no complete and unified preoperative standards of gastric cancer staging have been applied clinically. The results of our study show that the combination of EUS and PET-CT is an ideal madality in the preperative staging of gastric cancer. However, it should also be noted that many problems remain to be solved, such as the high cost of PET-CT and limited number of patients. Further study is required.

\section{Acknowledgments}

This study is supported by the Shanghai Natural Science Foundation of China (NO. 11ZR1429000)

\section{Conflict of Interest}

The authors declare no conflict of interest.

\section{References}

Akashi, K., Yanai, H., Nishikawa, J., Satake, M., Fukagawa, Y., Okamoto, T. \& Sakaida, I. (2006) Ulcerous change decreases the accuracy of endoscopic ultrasonography diagnosis for the invasive depth of early gastric cancer. Int. J. Gastrointest. Cancer, 37, 133-138.

Bhandari, S., Shim, C.S., Kim, J.H., Jung, I.S., Cho, J.Y., Lee, J.S., Lee, M.S. \& Kim, B.S. (2004) Usefulness of three-dimensional, multidetector row CT (virtual gastroscopy and multiplanar reconstruction) in the evaluation of gastric cancer: a comparison with conventional endoscopy, EUS, and histopathology. Gastrointest. Endosc., 59, 619-626.

Catalano, M.F., Sivak, M.V.Jr., Rice, T., Gragg, L.A. \& Van Dam, J. (1994) Endosonographic features predictive of lymph node metastasis. Gastrointest. Endosc., 40, 442-446.

Chen, C.H., Yang, C.C. \& Yeh, Y.H. (2002) Preoperative staging of gastric cancer by endoscopic ultrasound: the prognostic usefulness of ascites detected by endoscopic ultrasound. $J$. Clin. Gastroenterol., 35, 321-327.

Chen, C.Y., Hsu, J.S., Wu, D.C., Kang, W.Y., Hsieh, J.S., Jaw, T.S., Wu, M.T. \& Liu, G.C. (2007) Gastric cancer: preoperative local staging with 3D multi-detector row CT - correlation with surgical and histopathologic results. Radiology, 242, 472-482.

Dux, M., Richter, G.M., Hansmann, J., Kuntz, C. \& Kauffmann, G.W. (1999) Helical hydro-CT for diagnosis and staging of gastric carcinoma. J. Comput. Assist. Tomogr., 23, 913-922.

Ganpathi, I.S., So, J.B. \& Ho, K.Y. (2006) Endoscopic ultrasonography for gastric cancer: does it influence treatment? Surg. Endosc., 20, 559-562.

Gotoda, T. (2006) Endoscopic resection of early gastric cancer: the Japanese perspective. Curr. Opin. Gastroenterol., 22, 561569.

Greenberg, J., Durkin, M., Van, Drunen, M. \& Aranha, G.V. (1994) Computed tomography or endoscopic ultrasonography in preoperative staging of gastric and esophageal tumors. Surgery, 116, 696-701.

Hizawa, K., Iwai, K., Esaki, M., Matsumoto, T., Suekane, H. \& Iida, M. (2002) Is endoscopic ultrasonography indispensable in assessing the appropriateness of endoscopic resection for gastric cancer? Endoscopy, 34, 973-978.

Kamangar, F., Dores, G.M. \& Anderson, W.F. (2006) Patterns of cancer incidence, mortality, and prevalence across five continents: defining priorities to reduce cancer disparities in different geographic regions of the world. J. Clin. Oncol., 24, 2137-2150. 
Kwee, R.M. \& Kwee, T.C. (2007) Imaging in local staging of gastric cancer: a systematic review. J. Clin. Oncol., 25, 21072116.

Martin, R.C., Jaques, D.P., Brennan, M.F. \& Karpeh, M. (2002) Achieving RO resection for locally advanced gastric cancer: is it worth the risk of multiorgan resection? J. Am. Coll. Surg., 194, 568-577.

Meltzer, C.C., Luketich, J.D., Friedman, D., Charron, M., Strollo, D., Meehan, M., Urso, G.K., Dachille, M.A. \& Townsend, D.W. (2000) Whole-body FDG positron emission tomographic imaging for staging esophageal cancer comparison with computed tomography. Clin. Nucl. Med., 25, 882-887.

O'Reilly, S. \& Forastiere, A.A. (1995) Is surgery necessary with multimodality treatment of oesophageal cancer. Ann. Oncol., 6, 519-521.

Parkin, D.M., Bray, F., Ferlay, J. \& Pisani, P. (2002) Global cancer statistics, 2002. CA. Cancer J. Clin., 55, 74-108.

Polkowski, M., Palucki, J., Wronska, E., Szawlowski, A., Nasierowska-Guttmejer, A. \& Butruk, E. (2004) Endosonography versus helical computed tomography for locoregional staging of gastric cancer. Endoscopy, 36, 617-623.

Sohn, K.M., Lee, J.M., Lee, S.Y., Ahn, B.Y., Park, S.M. \& Kim, K.M. (2000) Comparing MR imaging and CT in the staging of gastric carcinoma. AJR. Am. J. Roentgenol., 174, 15511557.

Terry, M.B., Gaudet, M.M. \& Gammon, M.D. (2002) The epidemiology of gastric cancer. Semin. Radiat. Oncol., 12, 111-127.

Townsend, D.W. \& Beyer, T. (2002) A combined PET/CT scanner: the path to true image fusion. Br. J. Radiol., 75, S24-30.

Tsendsuren, T., Jun, S.M. \& Mian, X.H. (2006) Usefulness of endoscopic ultrasonography in preoperative TNM staging of gastric cancer. World J. Gastroenterol., 12, 43-47.

Walker, A.J., Spier, B.J., Perlman, S.B., Stangl, J.R., Frick, T.J., Gopal, D.V., Lindstrom, M.J., Weigel, T.L. \& Pfau, P.R. (2011) Integrated PET/CT fusion imaging and endoscopic ultrasound in the pre-operative staging and evaluation of esophageal cancer. Mol. Imaging Biol., 13, 166-171.

Willis, S., Truong, S., Gribnitz, S., Fass, J. \& Schumpelick, V. (2000) Endoscopic ultrasonography in the preoperative staging of gastric cancer: accuracy and impact on surgical therapy. Surg. Endosc., 14, 951-954.

Ziegler, K., Sanft, C., Zimmer, T., Zeitz, M., Felsenberg, D., Stein, H., Germer, C., Deutschmann, C. \& Riecken, E.O. (1993) Comparison of computed tomography, endosonography, and intraoperative assessment in TN staging of gastric carcinoma. Gut, 34, 604-610. 